\title{
Coherence Time Extension by Large-Scale Optical Spin Polarization in a Rare-Earth Doped Crystal
}

\author{
Sacha Welinski®, ${ }^{1, *}$ Alexey Tiranov $\odot,{ }^{2, \dagger}$ Moritz Businger, ${ }^{2}$ Alban Ferrier $\odot,{ }^{1,3}$ \\ Mikael Afzelius, ${ }^{2}$ and Philippe Goldner, ${ }^{1, \$}$ \\ ${ }^{1}$ Chimie ParisTech, PSL University, CNRS, Institut de Recherche de Chimie Paris, 75005 Paris, France \\ ${ }^{2}$ Département de Physique Appliquée, Université de Genève, CH-1211 Genève, Switzerland \\ ${ }^{3}$ Sorbonne Universités, Faculté des Sciences et Ingénierie, UFR 933, 75005 Paris, France
}

(Received 10 December 2019; revised 2 July 2020; accepted 27 July 2020; published 16 September 2020)

\begin{abstract}
Optically addressable spins are actively investigated in quantum communication, processing, and sensing. Optical and spin coherence lifetimes, which determine quantum operation fidelity and storage time, are often limited by spin-spin interactions, which can be decreased by polarizing spins. Spin polarization can be achieved using optical pumping, large magnetic fields, or mK-range temperatures. Here, we show that optical pumping of a small fraction of ions with a fixed-frequency laser, coupled with spin-spin interactions and spin diffusion, leads to substantial spin polarization in a paramagnetic rare-earth doped crystal, ${ }^{171} \mathrm{Yb}^{3+}: \mathrm{Y}_{2} \mathrm{SiO}_{5}$. Indeed, more than $90 \%$ spin polarization has been achieved at $2 \mathrm{~K}$ and zero magnetic field. Using this spin polarization mechanism, we further demonstrate an increase in optical coherence lifetime from $0.3 \mathrm{~ms}$ to $0.8 \mathrm{~ms}$, due to a strong decrease in spin-spin interactions. This effect opens the way to new schemes for obtaining long optical and spin coherence lifetimes in various solid-state systems such as ensembles of rare-earth ions or color centers in diamond, which are of interest for a broad range of quantum technologies.
\end{abstract}

DOI: 10.1103/PhysRevX.10.031060

Subject Areas: Materials Science, Optics,

Quantum Information

\section{INTRODUCTION}

Systems with both spin and optical transitions offer a range of functionalities for quantum technologies [1]. They allow storage and entanglement of photonic quantum states for quantum communications [2,3], interfacing processing nodes with optical networks for distributed quantum computing [4], or efficient detection for quantum sensing [5]. Key parameters in these centers are the coherence lifetimes of optical and spin transitions, which affect storage time, operation fidelity, and sensitivity. In the solid state, major sources of perturbation to quantum states are due to magnetic fluctuations in the center environment that couple to transitions through magnetic dipole-dipole interactions. This magnetic noise is often due to flips of electron

\footnotetext{
*Present address: Department of Electrical Engineering, Princeton University, Princeton, New Jersey 08544, USA.

${ }^{\dagger}$ Present address: The Niels Bohr Institute, University of Copenhagen, DK-2100 Copenhagen $\varnothing$, Denmark.

"philippe.goldner@chimieparistech.psl.eu

Published by the American Physical Society under the terms of the Creative Commons Attribution 4.0 International license. Further distribution of this work must maintain attribution to the author(s) and the published article's title, journal citation, and DOI.
}

or nuclear spins carried by the host material atoms [6], impurities or defects [7], or the centers of interest themselves [8,9]. Considerable work has been devoted to engineering these ensembles, known as the spin bath, in order to reduce their detrimental effect on coherence lifetimes. This work includes isotope purification to eliminate elements with nonzero spins $[10,11]$ and spin polarization. The latter can be obtained by application of high magnetic fields [12,13], very low temperatures [14], or optical pumping [15]. Another approach is to decouple the optically addressable spins from the bath by using clock transitions that are insensitive to magnetic-field fluctuations at first order [16-18] or by filtering out bath fluctuations by dynamical techniques $[19,20]$. Although they can be very efficient, these methods may be complex to implement.

Here, we show that optical pumping (OP) of a very small fraction $(\approx 0.5 \%)$ of spins in an ensemble can lead, through spin diffusion, to a polarization of this ensemble larger than $90 \%$. Furthermore, this large-scale change in spin populations can be tuned to strongly decrease spin-spin interactions and, in turn, extend optical coherence lifetimes close to the radiative limit. This process, which we call diffusion enhanced optical pumping (DEOP), is illustrated in Fig. 1(a). A narrow laser optically pumps a small subset of spins randomly located in the laser excitation volume [labeled "optical spins" in Fig. 1(a), left]. The optical spins 
(a) Thermal spin $\circ$ Optical spin $\bullet$ Polarized spin $\circ$
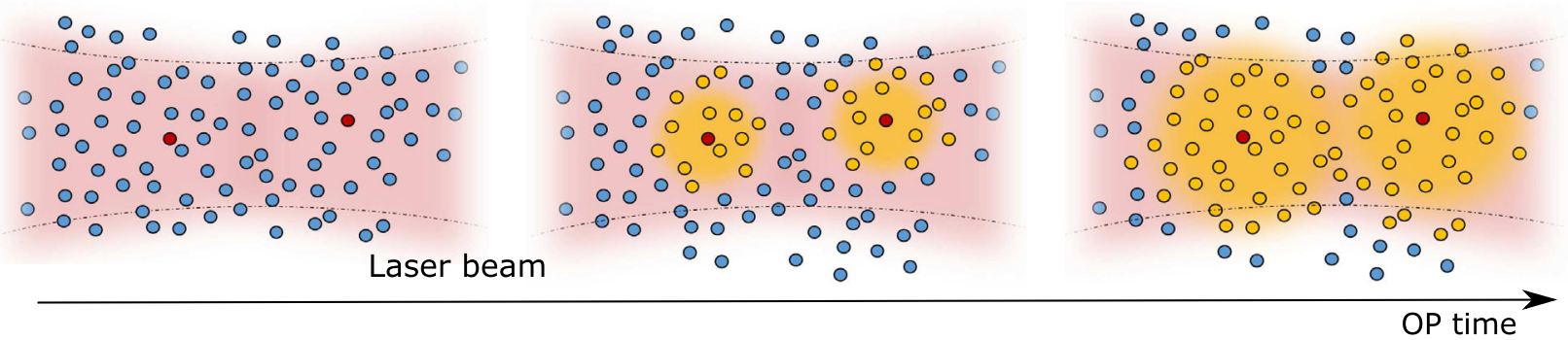

(b)

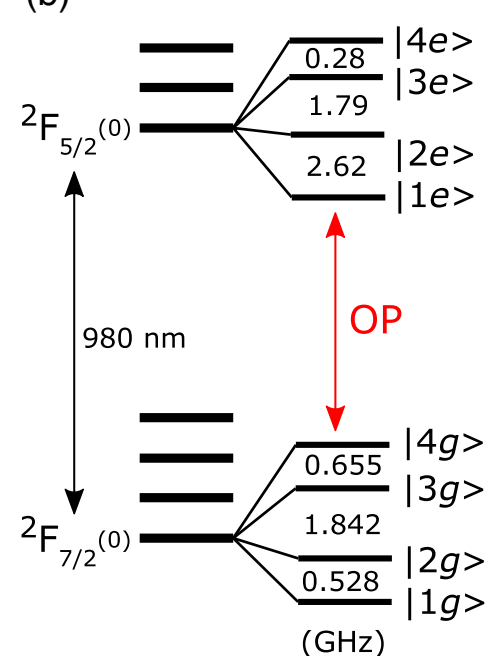

(c)

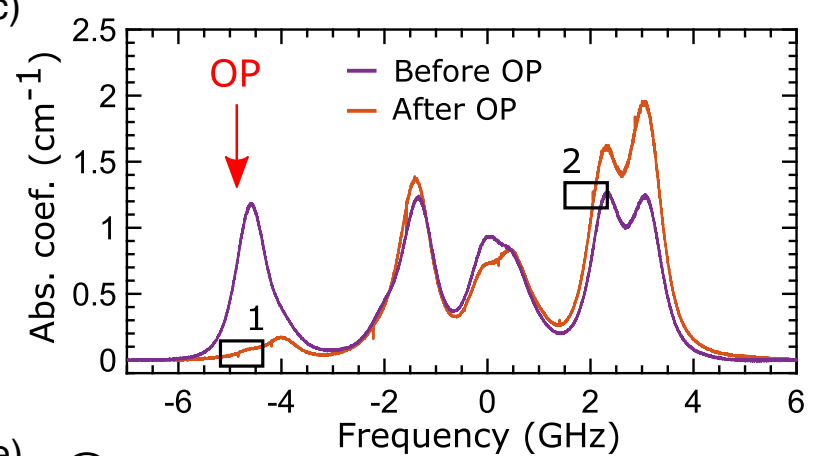

(d)

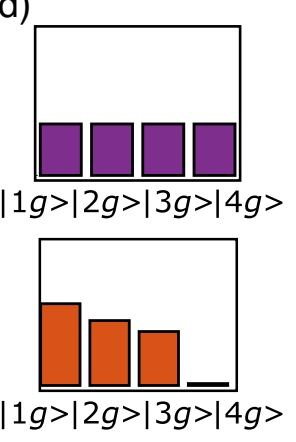

(e)

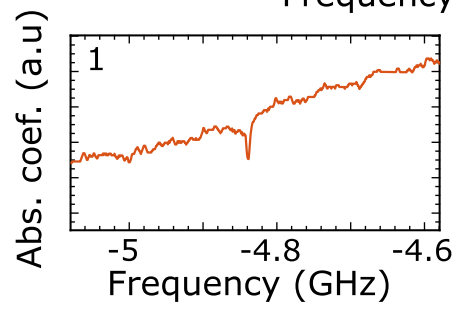

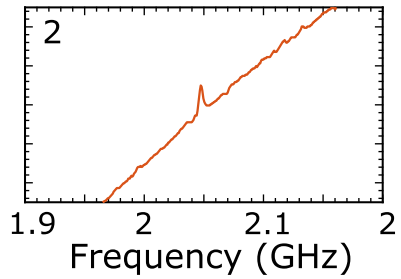

FIG. 1. Diffusion enhanced optical pumping (DEOP) in ${ }^{171} \mathrm{Yb}^{3+}: \mathrm{Y}_{2} \mathrm{SiO}_{5}$. (a) DEOP mechanism: Optically pumped spins (red circles) initially surrounded by spins in thermal equilibrium (blue circles) gradually polarize neighboring spins and spins that are further apart (yellow circles) through flip-flop interactions. (b) Energy diagram showing the ${ }^{171} \mathrm{Yb}^{3+}$ hyperfine structure. (c) Absorption spectrum without (purple) and after $20 \mathrm{~s}$ of optical pumping (orange). The OP is along the $|4 g\rangle \rightarrow|1 e\rangle$ transition [red arrow in panels (b) and (c)]. (d) Ground-state spin populations $k_{i g}$, normalized by thermal equilibrium values, over the volume addressed by the laser without OP (top, purple, $k_{i g}=1$ ) and after OP (bottom, orange, $k_{1 g}, k_{2 g}, k_{3 g}, k_{4 g}=1.67 \pm 0.30,1.28 \pm 0.36$, $1.01 \pm 0.12,0.04 \pm 0.04)$. (e) Enlarged regions 1 and 2 in panel (c) showing a narrow hole at the laser frequency $(|4 g\rangle \rightarrow|1 e\rangle)$ and a corresponding antihole $(|2 g\rangle \rightarrow|3 e\rangle)$.

exchange energy with neighboring spins through flip-flop processes. The latter are initially in thermal equilibrium ("thermal spins"), but because they interact with the strongly polarized optical spins, they become polarized as well ("polarized spins"), as shown in Fig. 1(a), center. The spin polarization gradually diffuses over the whole optically excited volume through further flip-flop interactions between polarized and thermal spins [Fig. 1(a), right]. As shown in this article, DEOP allows coherence lifetime extension by creating specific, highly out-ofequilibrium, population distributions among spin levels, a feature that is difficult to obtain by using magnetic field, low temperature, or optical pumping alone.

We demonstrate DEOP in a rare-earth (RE) doped crystal, ${ }^{171} \mathrm{Yb}^{3+}: \mathrm{Y}_{2} \mathrm{SiO}_{5}$ (YSO). These materials, in which optical and spin coherence lifetime can reach up to ms and hours at low temperatures $[6,12,21]$, are actively investigated for spin-based quantum photonic applications ranging from quantum memories [2,22-25], processors [26], and single-photon sources $[27,28]$ to optical-microwave transducers $[29,30]$. Note that ${ }^{171} \mathrm{Yb}^{3+}: \mathrm{YSO}$, in which long coherence lifetimes have been shown for both optical and spin transitions at zero magnetic field [18], is particularly promising in these areas [31-34]. Using DEOP, we obtain spin polarizations greater than $90 \%$, a much stronger effect than previously reported in ruby [35] and in Refs. [36,37], where spin diffusion was suggested as a hole-broadening mechanism for $\mathrm{Eu}^{3+}$ and $\mathrm{Pr}^{3+}$. Our analysis is supported by extensive experimental data and modeling, which, in turn, enables us to predict conditions under which DEOP leads to high polarization. Next, thanks to its spin-ensemble tailoring capabilities, we use DEOP to reduce or enhance specific spin-spin interactions and, in particular, extend ${ }^{171} \mathrm{Yb}^{3+}$ optical coherence lifetimes $T_{2}$ to about $800 \mu \mathrm{s}$, a 2.5 -fold increase compared to thermal equilibrium. This value is the longest optical $T_{2}$ reported 
for any paramagnetic solid-state system at zero or very-low magnetic fields, which can be especially interesting for interfacing with superconducting qubits and resonators. DEOP uses a counterintuitive scheme that exploits interactions to ultimately control them and should be effective in other materials. It paves the way to applications of concentrated, optically active, spin ensembles such as multimode optical or microwave quantum memories and high sensitivity magnetic sensing.

\section{RESULTS}

Experiments were performed using a $10-\mathrm{ppm}^{171} \mathrm{Yb}^{3+}$ : $\mathrm{Y}_{2} \mathrm{SiO}_{5}$ (YSO) single-crystal sample (see the Appendix). Note that ${ }^{171} \mathrm{Yb}^{3+}$ has $1 / 2$ electron and nuclear spins, and the corresponding ground-state $\left[{ }^{2} \mathrm{~F}_{7 / 2}(0)\right]$ and excited-state $\left[{ }^{2} \mathrm{~F}_{5 / 2}(0)\right]$ hyperfine structures, for ions in site 2 , are presented in Fig. 1(b). The optical transition is centered at $978.854 \mathrm{~nm}$ (vac.). Because of anisotropic Zeeman and hyperfine interactions, all hyperfine levels are nondegenerate, and their states show completely symmetric superposition of electron and nuclear spin projections (see Supplemental Material [38], for the wave-function expressions). This configuration results in levels that are insensitive to magnetic field fluctuations at first order under zero external magnetic field. Coherence lifetimes of all transitions are thus significantly enhanced for very low magnetic fields, reaching up to $4 \mathrm{~ms}$ and $180 \mu \mathrm{s}$ for spin and optical transitions at $3 \mathrm{~K}$ [18].

\section{A. Optical pumping}

DEOP was studied at $2 \mathrm{~K}$. A narrow linewidth (about $1 \mathrm{MHz}$ ) laser excited the ${ }^{2} \mathrm{~F}_{7 / 2}(0) \rightarrow{ }^{2} \mathrm{~F}_{5 / 2}(0)$ transition for a few 10 s of seconds. The laser was then blocked for a few ms to let the excited-state population relax to the ground state and finally, with a reduced power, shone again on the sample and frequency scanned to determine the ${ }^{171} \mathrm{Yb}^{3+}$ absorption spectrum (see the Appendix). In many crystals, RE spins can be optically pumped at low temperature since excitation to the optical state and subsequent decay often result in population transfer between ground-state spin levels. Since the laser linewidth is usually much narrower than the RE optical inhomogeneous linewidth, optical pumping creates spectral holes and antiholes in transmission spectra, i.e., regions of low and high absorption that can be as narrow as twice the optical homogeneous linewidth [21,39].

In ${ }^{171} \mathrm{Yb}^{3+}$ :YSO, spectral hole burning is not the only phenomenon that occurs. Indeed, as shown in Fig. 1(c), after pumping for $20 \mathrm{~s}$ the $|4 g\rangle \leftrightarrow|1 e\rangle$ transition with a $1-\mathrm{MHz}$ linewidth laser, the whole $550-\mathrm{MHz}$ inhomogeneously broadened line vanished. This result means that essentially all spins in the sample volume addressed by the laser that were initially in the $|4 g\rangle$ state $\left(\approx 2 \times 10^{14}\right.$ spins $)$ have been transferred to other spin states, despite only $0.4 \%$ of these spins being optically excited. The fraction of excited ions is determined from the overlap between the absorption spectrum and the laser line shape (see the Appendix). An analysis of the absorption spectrum, based on previously determined energies and branching ratios of transitions between ground- and excited-state spin levels, allowed us to accurately determine ground-state spin-level populations (see Refs. [32,40] and Supplemental Material [38] for details). This analysis shows that only $4 \%$ of the initial population is left in $|4 g\rangle$ [Fig. 1(d)]. We also note in Figs. 1(c) and 1(e) that holes and antiholes can be observed, although with a low contrast, in the spectrum. The frequency positions of the holes and antiholes correspond to the pattern expected under spectral hole burning [32], ruling out spurious effects like large laser drifts during optical pumping.

As explained in the Introduction, we attribute this large spin polarization to energy exchanges by flip-flop interactions between ${ }^{171} \mathrm{Yb}^{3+}$ ground-state spins. This process leads to a diffusion of the population imbalance imposed by the optical pumping of a small fraction of the spins. RE ions are randomly distributed over the volume of the crystal, and their optical frequencies, determined by local strains, are not expected to be correlated with their location [41]. Ions resonant at a given optical frequency are therefore distributed over the volume addressed by the laser, which results, under optical pumping, in a macroscopic spatial spin population gradient and, in turn, population diffusion, as illustrated in Fig. 1(a) and Supplemental Material [38]. The observation of a decrease of the overall optical absorption also indicates the absence of strong correlation between optical and spin transition frequencies. Indeed, assuming that spins with close energies flip flop more rapidly, such a correlation would lead to a faster decrease of absorption around the pump frequency.

An important goal of this study is to quantify how the degree of polarization depends on the fraction of optically pumped ions, as well as the characteristic time required to reach that polarization. To this end, we vary the optical pumping frequency across the $|4 g\rangle \leftrightarrow|1 e\rangle$ transition and record absorption spectra for different pumping durations $\tau_{P}$. Figure 2(a) shows the region of the absorption spectrum corresponding to the $|4 g\rangle \leftrightarrow|1 e\rangle$ transition, centered at zero-frequency detuning, with a smaller contribution from the $|3 g\rangle \leftrightarrow|1 e\rangle$ transition at $+0.655 \mathrm{GHz}$. The laser frequency is set at $-0.17 \mathrm{GHz}$, as shown by the hole that appears on spectra recorded for pump durations $1 \leq \tau_{P} \leq 25 \mathrm{~s}$. When $\tau_{P}$ is increased, the whole inhomogeneously broadened absorption decreases, without change in shape, and reaches a plateau after about $\tau_{P}=30 \mathrm{~s}$. From the peak absorption coefficient, measured at $0 \mathrm{GHz}$ in Fig. 2(a), we deduce $k_{4 g}$, which is the level $|4 g\rangle$ population normalized by its value without pumping, i.e., at thermal equilibrium (see the Appendix and Supplemental Material [38]). The same experiment was repeated for different laser 
(a)

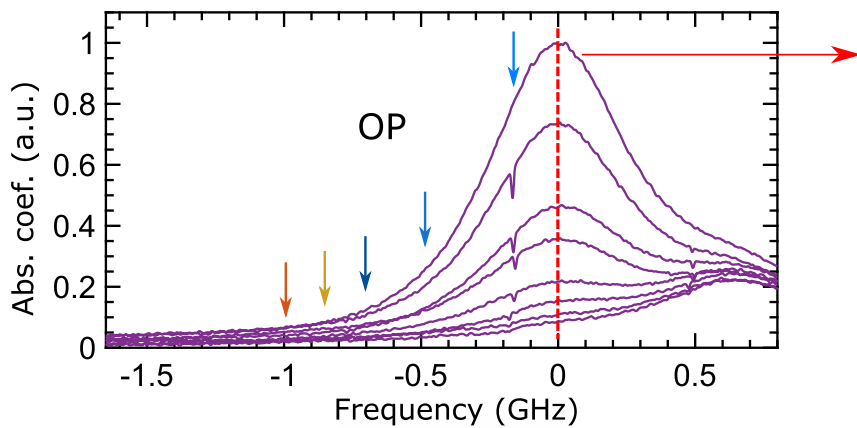

(c)

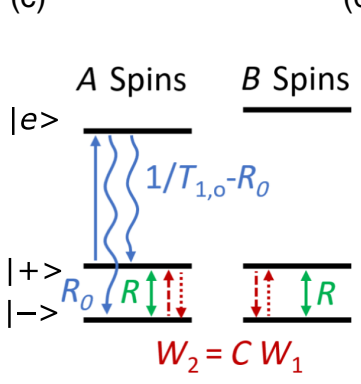

(d)

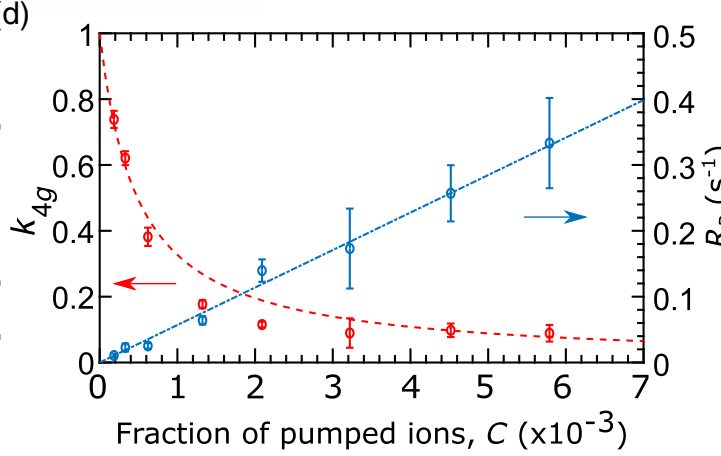

(b)

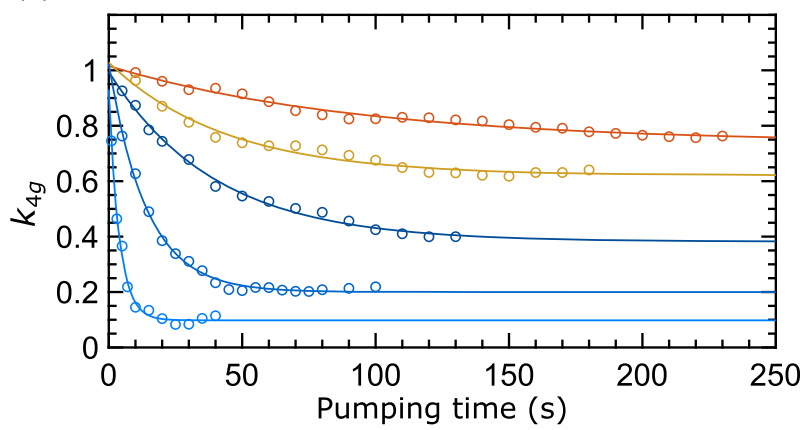

(e)

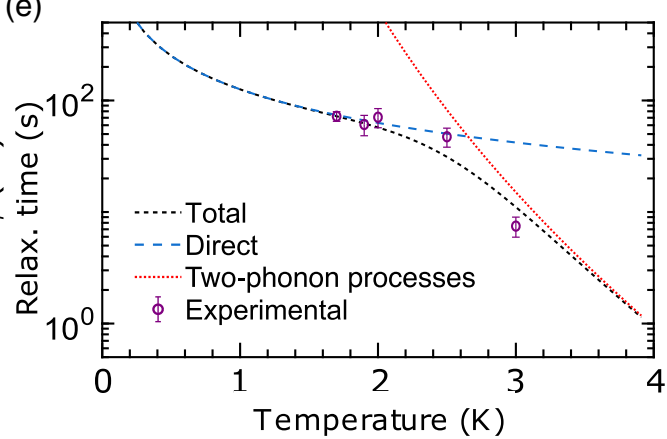

FIG. 2. Dynamics of DEOP and spin-lattice relaxation. (a) Absorption profile around the $|4 g\rangle \leftrightarrow|1 e\rangle$ transition after $0 \mathrm{~s}, 1 \mathrm{~s}, 3 \mathrm{~s}, 5 \mathrm{~s}$, $7 \mathrm{~s}, 10 \mathrm{~s}, 30 \mathrm{~s}$, and $40 \mathrm{~s}$ of OP at $-0.17 \mathrm{GHz}$ (light blue arrow). The whole inhomogeneously broadened absorption decreases when pumping duration increases. The laser creates a narrow spectral hole at $-0.17 \mathrm{GHz}$, which is clearly seen for pump duration between 1 and $7 \mathrm{~s}$. The small side hole at $+0.5 \mathrm{GHz}$ originates from the $|3 g\rangle$ level and should therefore appear as an antihole. This is explained by a fast relaxation between the $|4 g\rangle$ and $|3 g\rangle$ levels by flip-flop processes [32]. The absorption centered at $+0.655 \mathrm{MHz}$ corresponds to the $|3 g\rangle \leftrightarrow|1 e\rangle$ transition. (b) Normalized level $|4 g\rangle$ population $k_{4 g}$ as a function of OP duration for different laser frequencies shown in panel (a) by color-coded arrows. Solid lines are exponential fits to the data (see text). (c) Spin-1/2 model scheme (see text). (d) Level $|4 g\rangle$ normalized steady-state populations ( $k_{4 g}^{\infty}$, red circles) and polarization rate $\left(R_{P}\right.$, blue circles) as a function of the fraction of pumped ions (see text). Solid lines correspond to fits using a spin- $1 / 2$ model and rate equations. (e) Relaxation time $1 / R_{c}$ as a function of temperature deduced from absorption recovery after OP is stopped. The lines are fits using direct and two-phonon processes (see text). All error bars correspond to a $95 \%$ confidence interval.

frequencies, shown by the arrows in Fig. 2(a), which effectively reduce the fraction of optically pumped spins. The corresponding variations of $k_{4 g}$ are displayed in Fig. 2(b).

As the laser moves away from the peak absorption of the $|4 g\rangle \leftrightarrow|1 e\rangle$ transition, $k_{4 g}$ decreases more slowly as a function of $\tau_{P}$ and plateaus at a higher value. We find that it could be well fitted by an exponential expression of the form $k_{4 g}=\exp \left(-R_{P} \tau_{P}\right)+k_{4 g}^{\infty}$.

Figure 2(d) shows the variation of the steady-state population $k_{4 g}^{\infty}$ and the polarization rate $R_{P}$ as a function of the fraction $C$ of optically pumped ions, determined from the overlap between the absorption spectrum and laser line shape (see the Appendix). The variation of $R_{P}$ and $k_{4 g}^{\infty}$ can be understood in the following way: When $C$ decreases, each pumped spin has to polarize a larger number of nonpumped spins to reach a given $k_{4 q}$ value; this process slows down the overall spin diffusion and decreases the polarization rate $R_{P}$. The degree of achievable spin polarization is limited by the interaction of individual spins with the phonon bath, so-called spin lattice relaxation (SLR), which will counteract DEOP. Hence, the steady-state population $k_{4 g}^{\infty}$ is determined by the balance between SLR and spin diffusion rates, such that a smaller $R_{P}$ (and thus $C$ ) implies a larger $k_{4 g}^{\infty}$. At the highest fraction of pumped ions, $C=5.8 \times 10^{-3}, k_{4 g}^{\infty}=0.08 \pm 0.02$, and $1 / R_{P}=3 \pm 0.6 \mathrm{~s}$, which increases to $k_{4 g}^{\infty}=0.74 \pm 0.02$ and $1 / R_{P}=91 \pm 27 \mathrm{~s}$ for $C=2 \times 10^{-4}$, the lowest value investigated. Even by pumping such a small fraction of ions, $25 \%$ of $|4 g\rangle$ spins are transferred to another level, showing the efficiency of DEOP in this system.

The data in Fig. 2(d) suggest that $R_{P}$ depends linearly on $C$, whereas $k_{4 g}^{\infty}$ varies as $1 / C$. This can be accounted for by a simple model that treats ${ }^{171} \mathrm{Yb}^{3+}$ ions as an ensemble of $1 / 2$ spins divided into two groups: The $A$ spins are optically pumped to their ground state; the $B$ spins are not pumped but are expected to polarize to their lower state through DEOP [Fig. 2(c)]. Therefore, $C$ is the ratio between $A$ - and $B$-spin concentrations. We use rate equations to describe the individual relaxations as well as 
the flip-flop processes between $A$ and $B$ spins (see Supplemental Material [38] for details). They can be solved analytically, leading to

$$
\begin{gathered}
k_{4 g}^{\infty} \equiv \frac{p_{B}^{+, \infty}}{p_{B}^{+, e q}} \approx \frac{2}{2+C \beta_{\mathrm{ff}} / \beta_{o}}, \\
R_{P} \approx R\left(2+C \beta_{\mathrm{ff}} / \beta_{o}\right),
\end{gathered}
$$

where $p_{B}^{+, \infty}$ is the $B$-spin upper state population at a steady state, $p_{B}^{+, \text {eq }}$ is its thermal equilibrium value, and $R$ is the spin-lattice relaxation rate. These expressions indeed have the correct dependence on $C$ with respect to experimental observations. Note that $\beta_{\mathrm{ff}}=W_{1} /\left(W_{1}+R_{o}\right)$ and $\beta_{o}=R / R_{o}$, where $R_{o}$ is the $A$-spin effective optical pumping rate [Fig. 2(c)]. Here, $W_{1}$ is the relaxation rate of the $A$ spins by flip-flop interactions with the $B$ spins, and $W_{2}=C W_{1}$ is the relaxation rate of the $B$ spins by flip-flop interactions with the $A$ spins.

As shown in Fig. 2(d), reasonable agreement is obtained when fitting experimental $k_{4 g}^{\infty}$ and $R_{P}$ using Eqs. (1) and (2), which indicates that a two-level system can indeed be used to model ${ }^{171} \mathrm{Yb}^{3+}$ under these DEOP conditions. Flip-flop rates can be evaluated theoretically and give values that are reasonably consistent with experimental ones (see Supplemental Material [38]). This analysis shows that using a two-level model is possible due to the fast flipflop interactions that occur within the $|1 g\rangle-|2 g\rangle$ and $|3 g\rangle-|4 g\rangle$ pairs of levels (see Supplemental Material [38]). In this case, each pair of levels can be grouped and considered as one level, leading to an effective $1 / 2$ system. Flip-flop interactions within others pairs of levels, like $|4 g\rangle-|2 g\rangle$, are much slower. Here, $W_{1}$ corresponds to these slow rates, as they are found to be the limiting interactions for $B$-spin polarization. With the additional assumption $R_{o} \gg W_{1}$, the fits in Fig. 2(d) give $W_{1}=$ $57 \pm 5 \mathrm{~s}^{-1}$ and $R=(1.4 \pm 0.4) \times 10^{-2} \mathrm{~s}^{-1}$. We estimate $R_{o}=384 \mathrm{~s}^{-1}$ from the excited-state lifetime and optical branching ratios, and $W_{1}=13 \mathrm{~s}^{-1}$ from narrow-hole decays (see Supplemental Material [38]). These qualitative agreements support our simple spin- $1 / 2$ level-rate equation approach. However, we expect that when transitions connecting different ground-state levels are simultaneously pumped (see Sec. II B), a more complex four-level modeling is necessary.

Finally, we recorded the $|4 g\rangle \leftrightarrow|1 e\rangle$ absorption spectra at different delays after DEOP. As in the previous experiments, the line shape did not change while the initial absorption was gradually recovered, and the peak absorption coefficient allowed us to monitor level $|4 g\rangle$ population over all the spins in the volume addressed by the laser. Since flip-flop interactions do not change the overall level populations, the recovery rate $R_{c}$, obtained by an exponential fit to the data, corresponds to the SLR rate. This result is confirmed by the $R_{c}$ temperature dependence, which can be well modeled by a sum of a direct process and two-phonon processes, with parameters consistent with previous studies at higher magnetic field [33], as detailed in the Supplemental Material [38] [Fig. 2(e)]. At $2 \mathrm{~K}$, the temperature used for DEOP experiments, $R_{c}=1 /(72 \mathrm{~s})=1.4 \times 10^{-2} \mathrm{~s}^{-1}$, is in qualitative agreement with the fitted value $2 R=(2.8 \pm 0.8) \times 10^{-2} \mathrm{~s}^{-1}$.

\section{B. Optical coherence}

We next investigate optical coherence lifetimes $T_{2, o}$ under DEOP. This investigation was motivated by several studies that have shown that flipping ground-state spins of paramagnetic RE can be a major source of magnetic noise and therefore cause dephasing to RE optical and spin transitions $[8,33]$. This dephasing can be reduced by inducing strong spin polarization under large magnetic field and/or ultralow temperatures, by broadband optical pumping, or by using excited-state spins $[13,14,30,42,43]$. As shown in the previous section, DEOP also induces large-scale spin polarization and could therefore achieve similar effects.

Optical coherence lifetimes were measured for the $|4 g\rangle \leftrightarrow|1 e\rangle$ transition under several DEOP conditions. In a first series of measurements, DEOP was performed with a laser set at $-1.44 \mathrm{GHz}$ in the spectrum displayed in Fig. 3(a). At this frequency, some ions are pumped along the $|1 g\rangle \leftrightarrow|1 e\rangle$ transition and others along the $|3 g\rangle \leftrightarrow|2 e\rangle$ one because of the overlap between these inhomogeneously broadened lines. This process results in progressively pumping away the populations of the $|1 g\rangle$ and $|3 g\rangle$ levels when the DEOP duration is increased [Fig. 3(a)]. After $10 \mathrm{~s}$, nearly all the initial populations of these two levels have been transferred to $|4 g\rangle$ and $|2 g\rangle$. A second DEOP configuration was studied, setting the laser frequency at $+2.67 \mathrm{GHz}$ [Fig. 3(c)]. In this case, the optical excitation is resonant with the four optical transitions $|1 g\rangle \leftrightarrow|3 e\rangle$, $|1 g\rangle \leftrightarrow|4 e\rangle,|2 g\rangle \leftrightarrow|3 e\rangle$, and $|2 g\rangle \leftrightarrow|4 e\rangle$. As a result, the states $|1 g\rangle$ and $|2 g\rangle$ are now empty, and $|3 g\rangle$ and $|4 g\rangle$ are filled [Fig. 3(b)]. These two configurations highlight the versatility of DEOP, which allows polarizing spins in different levels and not only in the lowest energy one, as would result from using large magnetic fields or low temperatures.

For each laser frequency and DEOP duration, the optical coherence time of the $|4 g\rangle \leftrightarrow|1 e\rangle$ transition was measured with photon echoes (see the Appendix). The echo decays obtained by varying the delay between the excitation and rephasing pulses are displayed in Fig. 3(c). Decay rates show large variations as a function of DEOP conditions, and the corresponding coherence lifetimes $T_{2, o}$, obtained by single exponential fits, are gathered in Fig. 3(d). Without DEOP, $T_{2, o}=278 \pm 20 \mu \mathrm{s}$ or $\Gamma_{h, o}=$ $1 / \pi T_{2, o}=1.1 \pm 0.1 \mathrm{kHz}$. It reaches $782 \pm 30 \mu \mathrm{s}$ after $10 \mathrm{~s}$ of DEOP, which empties the $|1 g\rangle$ and $|3 g\rangle$ levels 

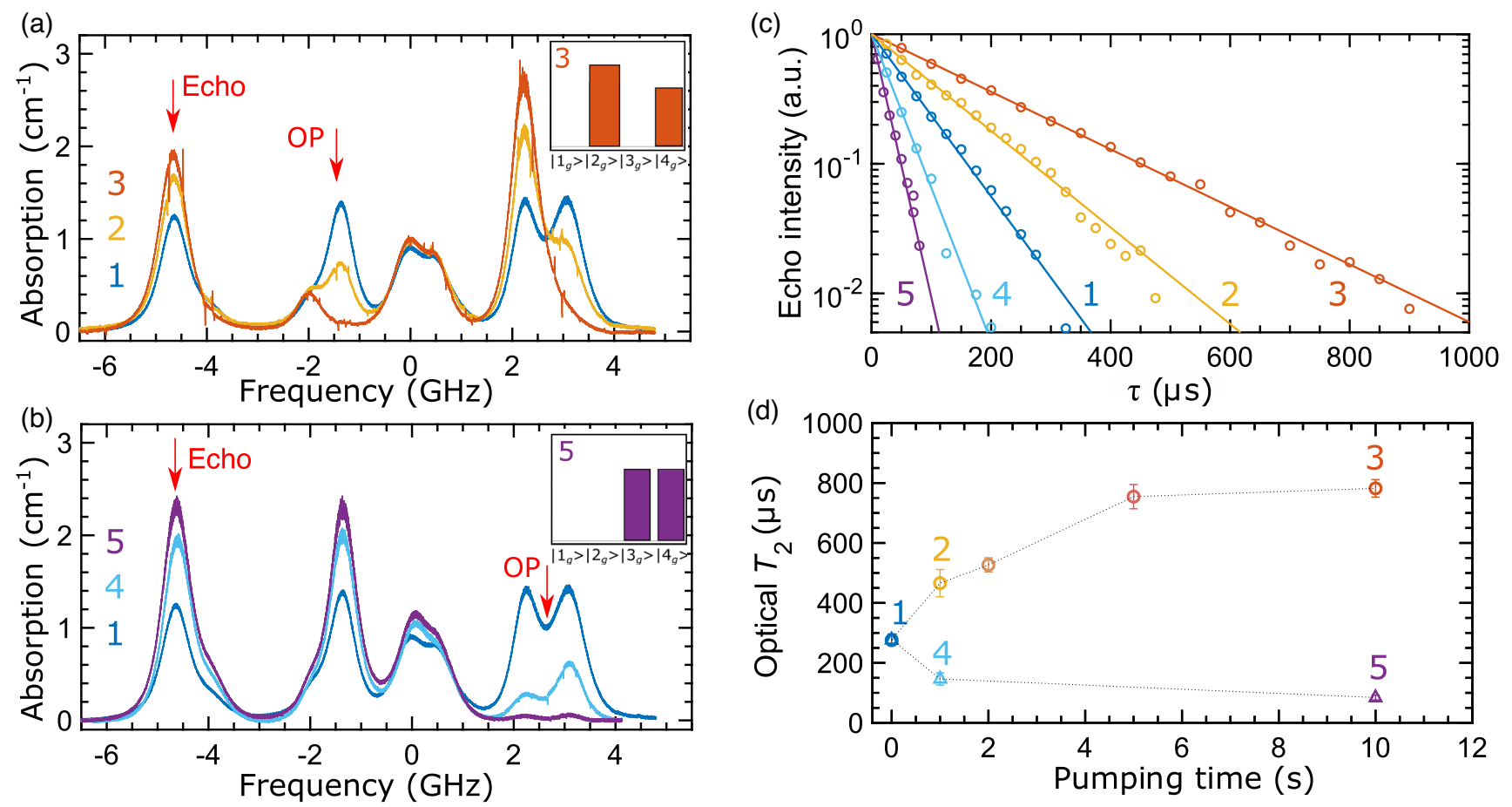

FIG. 3. Optical coherence lifetime under DEOP. (a) Absorption spectra after 0, 1, and $10 \mathrm{~s}$ of OP at -1.44 GHz. Inset: Normalized ground-state populations $k_{1 g}, k_{2 g}, k_{3 g}, k_{4 g}=0.02 \pm 0.02,2.30 \pm 0.12,0.02 \pm 0.02,1.70 \pm 0.06$. (b) Absorption spectra after 0, 1, and $10 \mathrm{~s}$ of $\mathrm{OP}$ at $+2.67 \mathrm{GHz}$. Inset: Normalized ground-state populations $k_{1 g}, k_{2 g}, k_{3 g}, k_{4 g}=0.02 \pm 0.02,0.02 \pm 0.02,1.95 \pm 0.10$, $2.05 \pm 0.08$. (c) Photon echo decays under DEOP for the $|4 g\rangle \leftrightarrow|1 e\rangle$ transition measured at $-4.6 \mathrm{GHz}$ [arrow in panels (a) and (b)]. Decay colors and numbers correspond to spectra in panels (a) and (b); solid lines are exponential fits to the data. (d) Optical coherence times $T_{2, o}$ deduced from fits in panel (c) (color-coded). All error bars correspond to a 95\% confidence interval.

[Figs. 3(a) and 3(d)]. The corresponding homogeneous linewidth is $\Gamma_{h, o}=407 \pm 15 \mathrm{~Hz}$. This is the narrowest homogeneous linewidth reported at zero magnetic field for any RE, with the exception of non-Kramers $\mathrm{Eu}^{3+}: \mathrm{Y}_{2} \mathrm{SiO}_{5}$ in which linewidths less than $290 \mathrm{~Hz}$ have been measured [44]. However, $\mathrm{Eu}^{3+}$ only has nuclear degrees of freedom, and its ground-state nuclear hyperfine structure spans only 60 to $160 \mathrm{MHz}$ in this crystal, depending on the isotope [45]. This amount is about 20 times less than ${ }^{171} \mathrm{Yb}^{3+}$ ( $3 \mathrm{GHz}$ ), which, in addition, has $7 \times 10^{3}$ times stronger spin transition dipole moments $[18,46]$. Therefore, ${ }^{171} \mathrm{Yb}^{3+}$ is much better suited for interactions with microwave photons while showing comparable optical coherence lifetimes.

When DEOP is used to empty $|1 g\rangle$ and $|2 g\rangle$ levels, a very different result is observed: $T_{2, o}$ is strongly reduced, down to $84 \pm 8 \mu$ s, which is equivalent to a homogeneous linewidth of $\Gamma_{h, o}=3.8 \pm 0.4 \mathrm{kHz}$. This result is a factor of 10 difference, as compared to the first DEOP configuration, and about 3.5 times the value obtained without pumping. To the best of our knowledge, this work is the first demonstration of changes, and especially significant enhancement, in coherence lifetimes induced by optical pumping. It is especially significant for systems that should be used at low magnetic field, to take advantage of magnetic insensitive transitions [16], as in ${ }^{171} \mathrm{Yb}^{3+}: \mathrm{Y}_{2} \mathrm{SiO}_{5}$ here, or when constraints from other devices such as superconducting resonators are relevant.

Contributions to the $|4 g\rangle \leftrightarrow|1 e\rangle$ homogeneous linewidth can be expressed in terms of level $|4 g\rangle$ and $|1 e\rangle$ population lifetimes $T_{1}$ and pure dephasing $\Gamma_{\phi}$ as

$$
\Gamma_{h, o}=\frac{1}{2 \pi T_{1,4 g}}+\frac{1}{2 \pi T_{1,1 e}}+\Gamma_{\phi}
$$

The excited-state lifetime $T_{1,1 e}$ can be taken to be simply its radiative lifetime, $T_{1,1 e}=T_{1, o}=1.3 \mathrm{~ms}$, as SLR rates are on the same order as in the ground state (close hyperfine and crystal-field splittings [31,32]) and the spin flip-flop rates are negligible due to the low concentration of excited ions. Hence, $T_{1,1 e}$ is considered independent of DEOP. For the ground state, we also disregard SLR contributions to $T_{1,4 g}$, as the estimated SLR lifetime is approximately $2 / R_{c}=144 \mathrm{~s}$. However, the flip-flop rates can contribute to $\Gamma_{h, o}$ both directly through the $T_{1,4 g}$ lifetime and indirectly through the dephasing term $\Gamma_{\phi}$. The contribution to $\Gamma_{\phi}$ is then a spectral diffusion process, where flip-flop interactions in the ${ }^{171} \mathrm{Yb}^{3+}$ spin bath create a time-varying magnetic-field noise on the optically probed ion. 
Both the direct and indirect flip-flop contributions are expected to change through DEOP. Our calculations of the flip-flop rates between ions in different hyperfine states show that the highest rates are due to flip-flop interactions in between ions in $|1 g\rangle$ and $|2 g\rangle$, and $|3 g\rangle$ and $|4 g\rangle$, respectively (see Supplemental Material [38]). Hence, we expect that the flip-flop rate will strongly decrease when ions are pumped into states $|2 g\rangle$ and $|4 g\rangle$ using DEOP, as in Fig. 3(a). Conversely, we expect the flip-flop rate to strongly increase when ions are pumped into states $|3 g\rangle$ and $|4 g\rangle$ using DEOP, as in Fig. 3(b). This argument qualitatively explains the change in coherence time due to DEOP, as seen in Figs. 3(c) and 3(d). However, these data are not sufficient to distinguish between the direct (lifetime) and indirect (spectral diffusion) contributions to the coherence time.

To this end, we also perform spin coherence measurements on the $|3 g\rangle \leftrightarrow|4 g\rangle$ transition at $655 \mathrm{MHz}$, as described in the Supplemental Material [38]. For this process, we polarize a large fraction of the spins into either the $|1 g\rangle$ and $|2 g\rangle$ states, or the $|3 g\rangle$ and $|4 g\rangle$ states, respectively. In both case, the populations in these two levels are essentially the same. An indirect spectral diffusion contribution to the spin coherence lifetime would be roughly equal in both cases, as the flip-flop rates are expected to be the same for both cases (see flipflop calculations in Supplemental Material [38]). However, the direct lifetime contribution to the probed states $|3 g\rangle$ and $|4 g\rangle$ would strongly decrease when spins are polarized into $|1 g\rangle$ and $|2 g\rangle$ states, as the flip-flop probability of spins in both the $|3 g\rangle$ and $|4 g\rangle$ states would be reduced. Indeed, we observe a strong change in spin coherence lifetime for the two cases, going from $0.2 \pm 0.02$ to $2.5 \pm 0.1 \mathrm{~ms}$ as spins are polarized into $|1 g\rangle$ and $|2 g\rangle$ states.

The spin coherence measurements indicate that direct flip-flop lifetimes significantly contribute to the optical coherence lifetimes in 10-ppm doped ${ }^{171} \mathrm{Yb}^{3+}: \mathrm{Y}_{2} \mathrm{SiO}_{5}$. Strong spin polarization using DEOP into selected hyperfine states can strongly reduce this contribution, as well as indirect contributions, to optical and spin dephasing. DEOP therefore allows increasing optical and spin coherence lifetimes at the same time.

Figure 4 displays the variation of the optical homogeneous linewidth $\Gamma_{h, o}$ as a function of pumping time using data from the upper branch of Fig. 3(d). Contributions to $\Gamma_{h, o}$ are divided into three parts. The first two are included in the term $1 / 2 \pi T_{1,4 g}+\Gamma_{\phi}$ in Eq. (3), and the third corresponds to $1 / 2 \pi T_{1,1 e}$.

(1) Blue area in Fig. 4: Direct and indirect processes described above and related to ${ }^{171} \mathrm{Yb}^{3+}$ in site 2 . The plateau in $\Gamma_{h, o}$ observed for long pumping times clearly indicates that DEOP is able to reduce this contribution to values $(<15 \mathrm{~Hz})$ significantly lower than other contributions and, in particular, the radiative one (see below).

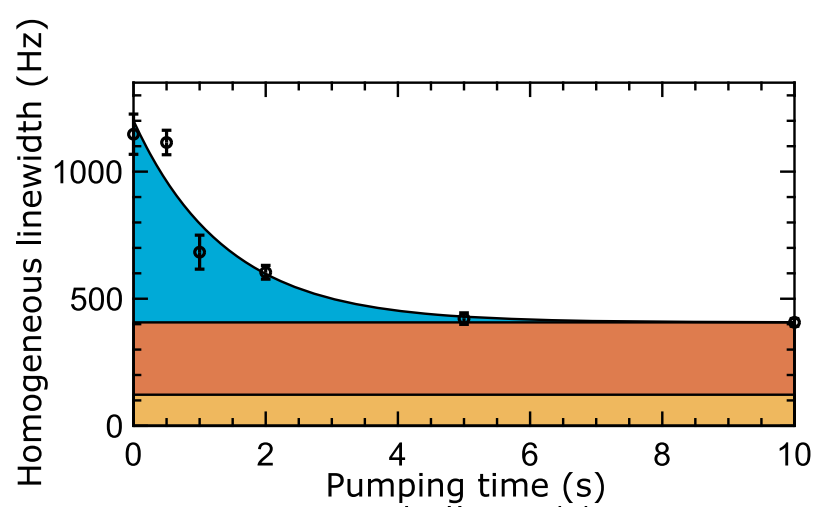

FIG. 4. Optical homogeneous linewidth $\Gamma_{h, o}$ of the $|4 g\rangle \leftrightarrow|1 e\rangle$ transition at $-4.6 \mathrm{GHz}$ as a function of pumping time under DEOP. Optical pumping is set at $-1.44 \mathrm{GHz}$ [see Fig. 3(a)]. The area under the curves represents contributions to $\Gamma_{h, o}$ from ${ }^{171} \mathrm{Yb}^{3+}$ in site 2 (blue), other centers including ${ }^{171} \mathrm{Yb}^{3+}$ in site 1 (orange), and the excited-state lifetime (yellow). The line drawn through experimental data points is a guide to the eye.

(2) Orange area: Indirect processes not influenced by DEOP causing a broadening of approximately $285 \mathrm{~Hz}$. Presumably, ${ }^{171} \mathrm{Yb}^{3+}$ ions in site 1, which represent $50 \%$ of the total $\mathrm{Yb}^{3+}$ concentration, cause the main part of this contribution through the second-order sensitivity of the optical transition to magnetic perturbations. Magnetic noise originating from ${ }^{89} \mathrm{Y}$ nuclear spins could also cause broadening through this mechanism. However, it is likely to be negligible as it contributes less than $60 \mathrm{~Hz}$ to the $\mathrm{Er}^{3+}: \mathrm{Y}_{2} \mathrm{SiO}_{5}$ optical transition, which has a firstorder sensitivity to magnetic perturbations [12].

(3) Yellow area: Excited-state lifetime contribution, $122 \mathrm{~Hz}$, which is the radiative limit.

This analysis suggests that $\Gamma_{h, o}$ could reach the radiative limit of $122 \mathrm{~Hz}$ within a few $10 \mathrm{~s}$ of $\mathrm{Hz}$ by strongly reducing flip-flop interactions between ${ }^{171} \mathrm{Yb}^{3+}$ ions in site 1 . This limit could be achieved by using DEOP on an appropriate transition, in the same way as shown here for ${ }^{171} \mathrm{Yb}^{3+}$ ions in site 2 , which would only require tuning a second laser to the relevant transition around $981.46 \mathrm{~nm}$ (vac) [31].

Although the above arguments can account for the general trends observed, a more detailed modeling and additional experiments are needed to precisely evaluate the processes affecting $T_{2, o}$. In particular, all ground-state flipflop interactions should be included in simulations, and their rates should be determined using spectral hole burning or other techniques. Further theoretical calculations of flipflop rates and frequency shifts caused by spin flips could also be very useful.

\section{DISCUSSION}

The spin- $1 / 2$ rate equation model [Eqs. (1) and (2) is convenient to estimate parameters for efficient DEOP, 
i.e., low remaining population in the $B$-spin upper level, $p_{B}^{+, \infty}$. First, low values of $\beta_{o}=R / R_{o}$ are required and therefore, a small SLR rate $R$ and/or strong optical pumping, i.e., larger $R_{o}$. The latter may be limited, as in our case, by the spontaneous emission rate and branching ratios, which in turn can be increased using optical nanocavities [27,28,47]. Small $R$ values can be achieved by lowering magnetic field and temperature [8], which is the case in our experiments, running at $2 \mathrm{~K}$ and zero magnetic field, giving $\beta_{o} \approx 3.6 \times 10^{-5}$ and polarizations over $90 \%$. However, SLR increases quickly with temperature or magnetic field, and Fig. 2(e) modeling predicts that at $4 \mathrm{~K}$ and zero field, DEOP polarizes only $11 \%$ of the spins. Large $\beta_{\mathrm{ff}}$ is also favorable and corresponds to strong flipflop interactions, i.e., large $W_{1}$. This result can be obtained with high spin concentration $c$ and lower inhomogeneous linewidth since $W_{1} \propto c^{2} / \Gamma_{\text {inh,spin }}$ [8]. In ${ }^{171} \mathrm{Yb}^{3+}: \mathrm{Y}_{2} \mathrm{SiO}_{5}$, it is worth noting that the spin linewidth is especially narrow at zero magnetic field, $\Gamma_{\text {inh,spin }}=1 \mathrm{MHz}$ [18], which increases flip-flop rates and gives $\beta_{\mathrm{ff}} \approx 0.13$. Finally, pumping a larger fraction of ions will result in stronger polarization. For example, the model indicates that increasing $C$ from the experimental value of $5.8 \times 10^{-3}$ to $2.8 \times 10^{-2}$ results in a decrease of $p_{B}^{+, \infty}$ from 0.04 to 0.01 , i.e., a $99 \%$ polarization. At the same time, $1 / R_{p}$ drops from 3.2 to $0.73 \mathrm{~s}$. Such an increase in $C$ could be obtained by scanning the laser over $25 \mathrm{MHz}$.

While frequency scanning over a small range can improve DEOP, obtaining high polarizations by optical pumping over a large frequency range, but without spin diffusion, may be much more difficult. We label the latter method LSOP for large-scan optical pumping in the following and take the experiment described in Fig. 1(c) as an example. Emptying the $|4 g\rangle$ level to $k_{4 g}^{\infty}<0.05$ without spin diffusion requires scanning the $|4 g\rangle \leftrightarrow|1 e\rangle$ transition (centered at $-4.5 \mathrm{GHz}$ ) essentially over its entire linewidth. As shown in Fig. 1(c), this process cannot be done without also pumping the $|3 g\rangle \leftrightarrow|1 e\rangle$ transition (centered at $-4 \mathrm{GHz}$ ) and therefore repopulating level $|4 g\rangle$ through the strong $|1 e\rangle \rightarrow|4 g\rangle$ relaxation (see Supplemental Material [38]). Since $|1 e\rangle$ can also relax to $|3 g\rangle$ while pumping on $|4 g\rangle \leftrightarrow|1 e\rangle$, it is possible, depending on optical pumping rates and scanning speed, that an equilibrium between the $|4 g\rangle$ and $|3 g\rangle$ populations takes place, leading to a lower polarization than DEOP. In general, because of overlapping optical transitions, pumping by scanning over broad frequency ranges tends to shuffle population between levels rather than result in high polarization.

DEOP experiments when more than one transition is optically pumped, as investigated in Sec. II B, are more difficult to analyze with a simple spin-1/2 model. Nonpumped spins will interact with several classes of optically pumped spins that are polarized in different levels.
This interaction can lead to high polarization, as we observed, but also to opposite population changes and, therefore, remaining populations in some pumped levels. In this respect, isolated optical transitions, like the $|4 g\rangle \leftrightarrow|1 e\rangle$ in ${ }^{171} \mathrm{Yb}^{3+}: \mathrm{Y}_{2} \mathrm{SiO}_{5}$, simplify pumping schemes and will appear for spin splittings at least comparable to the optical inhomogeneous linewidth. The previous discussion suggests a way to classify materials in relation to high ensemble polarization by DEOP:

(1) When optical transitions from different ground-state spin levels strongly overlap, the overall spin population cannot accumulate into a level that is not optically pumped. As a result, DEOP, as well as LSOP, only polarize a subset of spins. Materials that show optical inhomogeneous linewidths $\Gamma_{\text {inh }}$ much larger than spin splittings are therefore not good candidates for large-scale spin polarization by DEOP or LSOP. RE ions with nuclear spins only, such as ${ }^{151} \mathrm{Eu}^{3+}$, fall into this category, with $\Gamma_{\text {inh }}$ in the $\mathrm{GHz}$ range and spin gyromagnetic ratios of a few $\mathrm{MHz} / \mathrm{T}$ [21]. Another example are ensembles of $\mathrm{NV}^{-}$centers in diamond, with $\Gamma_{\text {inh }}$ of $100 \mathrm{~s}$ of $\mathrm{GHz}$ and a zero-field splitting of $3 \mathrm{GHz}$ [48]. DEOP in this system may still be possible, though, because of a strongly cycling transition that is, in effect, isolated from the other ones, even with spectral overlap [49].

(2) On the contrary, if an optical transition has no overlap with those originating from other groundstate spin levels, DEOP and LSOP can strongly polarize spin ensembles. Materials with very narrow optical inhomogeneous linewidths, and/or large $g$ factors, such as $\mathrm{Er}^{3+}: \mathrm{LiYF}_{4}$ [14], $\mathrm{Yb}^{3+}: \mathrm{YVO}_{4}$ [34], or $\mathrm{Er}^{3+}: \mathrm{Y}_{2} \mathrm{SiO}_{5}$ [50], can show such properties. LSOP, which does not rely on spin diffusion, may be advantageous over DEOP in this case, in terms of, e.g., polarization rate. However, it may require scanning a laser over large bandwidths, whereas a fixed-frequency laser is sufficient for DEOP; a strong magnetic field may also be necessary to split spin levels much more than $\Gamma_{\text {inh }}$, which in turn increases SLR through direct processes and could therefore be detrimental to high polarization. The latter effect can be especially important in paramagnetic RE ions [8].

(3) The intermediate regime, where transitions partially overlap, is where DEOP is the most effective. It corresponds to materials with optical inhomogeneous linewidths comparable to spin splittings. In the next paragraph, we list some systems of interest for quantum technologies that belong to this category and could behave as ${ }^{171} \mathrm{Yb}^{3+}$ :YSO under DEOP.

Inorganic crystals doped with RE or transition metal ions can exhibit narrow inhomogeneous optical lines, in the $\mathrm{GHz}$ range or below, and indeed, DEOP was observed in $\mathrm{Cr}^{3+}: \mathrm{Al}_{2} \mathrm{O}_{3}$ [35] and can also be seen in $\mathrm{Nd}^{3+}: \mathrm{YVO}_{4}$ as 
shown in Fig. 5 of Ref. [51], although it was not recognized as such in this work. It could also be observed in other RE ions of interest for applications in quantum technologies. As an example, we estimated the magnetic fields, SLR, and flip-flop rates necessary to achieve DEOP as efficiently as in ${ }^{171} \mathrm{Yb}^{3+}: \mathrm{Y}_{2} \mathrm{SiO}_{5}$ for $\mathrm{Er}^{3+}$ and $\mathrm{Nd}^{3+}$ ions in $\mathrm{Y}_{2} \mathrm{SiO}_{5}$ (see Table S III in Supplemental Material [38]). These materials have been investigated for quantum memories, optical to microwave quantum transducers, and quantum processors $[2,30,52-54]$. We first found that magnetic fields in the range $1-100 \mathrm{mT}$ are sufficient to partially remove transition overlap, thanks to $\Gamma_{\text {inh }}=0.2$ (6) $\mathrm{GHz}$ and $g=15$ (4) for $\mathrm{Er}^{3+}\left(\mathrm{Nd}^{3+}\right)$. Second, the analysis yields a maximum temperature $T_{\max }$ and minimum RE concentration $c_{\min }$, such that DEOP can be achieved when simultaneously $T<T_{\max }$ and $c>c_{\min }$. Values for $T_{\max }$ and $c_{\min }$ are found in reasonable ranges ( 0.8 to $2.6 \mathrm{~K}$ and 0.1 to $100 \mathrm{ppm}$ ), suggesting that DEOP could be an effective technique in these materials. At the same time, it also explains why previous experimental conditions may have prevented observing efficient DEOP. As an example, many holeburning experiments were carried out in $\mathrm{Nd}^{3+}: \mathrm{Y}_{2} \mathrm{SiO}_{5}$ (site 1) $[2,43]$ but at temperatures above $3 \mathrm{~K}$ and mostly under high magnetic field. This process increases SLR and reduces flip-flop rates, resulting in lower DEOP (see Table S III).

Paramagnetic RE with nonzero nuclear spins $(I \neq 0)$ that exhibit ground-state splittings of a few $\mathrm{GHz}$ at zero magnetic field such as ${ }^{167} \mathrm{Er}^{3+},{ }^{145} \mathrm{Nd}^{3+}$ or ${ }^{173} \mathrm{Yb}^{3+}$ could also achieve DEOP under similar conditions, assuming that spin relaxations are dominated by the electron part of the hyperfine wave functions. These isotopes have particular interest over the $I=0$ isotopes since they can achieve long coherence lifetimes as they can show optical and spin zero first-order Zeeman transitions at zero magnetic field, in the same way as ${ }^{171} \mathrm{Yb}^{3+}: \mathrm{Y}_{2} \mathrm{SiO}_{5}[18,55]$.

Applying DEOP to other spin systems that possess narrow optical transitions should also be possible, as shown in Table S IV in Supplemental Material [38]. Materials of interest include dopants like $\mathrm{Cr}$, Mo, or Se in silicon and $\mathrm{SiC}$, as well as Si-related color centers in diamond and $\mathrm{SiC}$. They are considered as promising candidates for optical quantum technologies such as spin-photon entanglement sources for long-distance communication and electronspin-based quantum processors [56,57]. The main difference with RE ions is shorter excited-state lifetimes, which result in polarization levels that essentially depend on spin population lifetime $T_{1}$ and center concentration [38]. The most promising systems are those with long $T_{1}$ and narrow $\Gamma_{\text {inh }}$, such as $\mathrm{Cr}^{4+}: \mathrm{SiC}$ and ${ }^{77} \mathrm{Se}^{+}: \mathrm{Si}$, the latter showing spin properties close to $\mathrm{RE}$ ions [58]. $\mathrm{Mo}: \mathrm{SiC}$ and $\mathrm{SiV}$ centers in $\mathrm{SiC}$ and diamond have larger $\Gamma_{\text {inh }}$ and would require stronger magnetic field to decrease overlap but also exhibit long spin $T_{1}$. Efficient DEOP could be observed in all these systems, provided concentrations allow sufficient spin-spin interactions (Table S IV). Such concentrated systems could also enable the implementation of quantum gates between spins for large-scale and/or long-distance quantum communication and processing.

As in the case for ${ }^{171} \mathrm{Yb}^{3+}: \mathrm{Y}_{2} \mathrm{SiO}_{5}$, DEOP could extend optical and/or spin coherence lifetimes of these other solidstate systems by reducing spin-spin interactions. Therefore, it allows keeping a high concentration of active species with low dephasing. DEOP can also provide large-scale spin initialization prior to processing and/or spectral tailoring. As an example, we achieved $96 \pm 1 \%$ polarization in the single $|4 g\rangle$ level of ${ }^{171} \mathrm{Yb}^{3+}: \mathrm{Y}_{2} \mathrm{SiO}_{5}$ (see Supplemental Material [38]). These features could be particularly useful for several applications based on ensembles. Absorptive quantum memories require high optical absorption and long coherence lifetimes [59]. While memory protocols that do not require spectral tailoring, like ROSE [60], SEMM [61], or RASE [62], could directly use DEOP, others, like AFC [63], require the creation of narrow optical features within the inhomogeneous linewidths. This may be more difficult to achieve in systems with efficient DEOP since spin diffusion will also limit hole lifetime and hence maximal optical depth. However, we note that in the example of ${ }^{171} \mathrm{Yb}^{3+}: \mathrm{Y}_{2} \mathrm{SiO}_{5}$, large polarization and coherence lifetime are obtained with a relatively small flip-flop rate of $13 \mathrm{~s}^{-1}$. Such a value could still allow for burning deep holes since the optical pumping rate, limited by the excited-state lifetime, is about 30 times higher. In addition, deep and narrow spectral holes could be created by fast coherent techniques, circumventing the hole lifetime limitation [64]. In systems with several groundstate spin levels, it may even be possible to perform spinwave storage in addition to spectral tailoring. A specific DEOP-based optical pumping and spin storage scheme for ${ }^{171} \mathrm{Yb}^{3+}$ :YSO is presented in the Supplemental Material [38], suggesting that DEOP is indeed suitable for longstorage-time, high-efficiency quantum memories. Another area where DEOP could be useful is quantum microwave to optical transduction, where high polarization enhances coupling to optical and microwave fields in off-resonant [29] and resonant [65] protocols. In the latter, extending optical and spin coherence lifetimes is also important and could be achieved by DEOP, which is further discussed for ${ }^{171} \mathrm{Yb}^{3+}: \mathrm{Y}_{2} \mathrm{SiO}_{5}$ in the Supplemental Material [38].

In conclusion, we have observed large-scale spin polarization under laser excitation at fixed frequency in a rareearth doped crystal, ${ }^{171} \mathrm{Yb}^{3+}: \mathrm{Y}_{2} \mathrm{SiO}_{5}$. This effect is explained by a combination of optical pumping and spin diffusion by flip-flop interactions that results in greater than $90 \%$ polarization for all spins in the sample volume addressed by the laser. Furthermore, the efficiency and versatility of the process are demonstrated by significantly increasing and decreasing optical coherence lifetimes $T_{2, o}$, depending on the pumping conditions. A value of $T_{2, o}$ up to approximately $800 \mu \mathrm{s}$ was recorded, the longest reported 
for a paramagnetic RE at zero magnetic field and in the range to that of nonparamgnetic RE. Given the other favorable optical and spin properties of ${ }^{171} \mathrm{Yb}^{3+}: \mathrm{Y}_{2} \mathrm{SiO}_{5}$, our results open the way to new designs for broadband and efficient quantum memories for light [40] or optical to microwave transducers. We expect this process to be efficient in other rare-earth doped crystals and concentrated systems of optically addressable spins. It could be used to tailor spin baths and therefore extend coherence lifetimes or initialize spins on a large scale, topics which are central to many quantum technologies.

\section{ACKNOWLEDGMENTS}

We would like to thank John Bartholomew for very useful suggestions to improve the manuscript. This project has received funding from the European Union's Horizon 2020 research and innovation programme under Grant Agreement No. 712721 (NanOQTech), the IMTO Cancer AVIESAN (Cancer Plan, C16027HS, MALT), the ANR MIRESPIN project, Grant No. ANR-19-CE470011 of the French Agence Nationale de la Recherche, and FNS Research Project No. 172590.

\section{APPENDIX: EXPERIMENTAL DETAILS}

\section{Sample}

The $\mathrm{Y}_{2} \mathrm{SiO}_{5}$ single crystal was doped with $10 \mathrm{ppm}$ of ${ }^{171} \mathrm{Yb}^{3+}$ (94\% isotopic purity; see Supplemental Material [38]) and grown by the Czochralski technique. It was cut along the extinction axis $b, D_{1}$ and $D_{2}$, with light propagating along the $b$ axis and polarized along $D_{2}$ for maximal absorption. The length of the sample along $b$ was $9.4 \mathrm{~mm} . \mathrm{Y}_{2} \mathrm{SiO}_{5}$ has a monoclinic structure and belongs to the $\mathrm{C}_{2 h}^{6}$ space group. $\mathrm{Yb}^{3+}$ can substitute $\mathrm{Y}^{3+}$ ions equally in their two sites of $C_{1}$ point symmetry.

\section{Experimental setup and optical pumping}

The sample was placed inside a liquid helium bath cryostat at $2 \mathrm{~K}$. Excitation was provided by a tunable single-mode diode laser (Toptica DL 100) with a spectral width of $1 \mathrm{MHz}$. The beam on the sample was weakly focused on the sample with a diameter of $1 \mathrm{~mm}$. All experiments were performed in transmission mode. Spectra were calibrated by recording the signal from a Toptica FPI 100 Fabry-Perot interferometer (1-GHz free spectral range). An acousto-optic modulator (AOM, AA Optoelectronics MT80) in a single-pass configuration was used to gate the laser. The detector we used was an amplified Si photodiode (Thorlabs PDA150A). The power during optical pumping and scans was $7 \mathrm{~mW}$ and $0.4 \mathrm{~mW}$, with frequency scans performed at a rate of $3 \mathrm{GHz} / \mathrm{ms}$. A delay of $10 \mathrm{~ms}$ was kept between optical pumping and scanning to let the excited-state population decay to the ground state. To probe absorption recovery, scans were performed at different delays after $20 \mathrm{~s}$ of optical pumping and at different temperatures.

The fraction of pumped ions were calculated from absorption spectrum using the formula

$$
C=\frac{2}{\pi} \frac{\Gamma}{\Gamma_{0}} \frac{\alpha}{\alpha_{0}}
$$

where $\alpha$ and $\alpha_{0}$ are absorption coefficients at the laser frequency and peak of the line, $\Gamma_{0}$ is the full width at half maximum, and $\Gamma$ is the pumped region spectral width. We have $\Gamma_{0}=550 \mathrm{MHz}$ [18] and $\Gamma \approx 5 \mathrm{MHz}$. The latter value corresponds to the hole observed on the absorption spectra and takes into account the effective laser linewidth on a timescale of the order of the excited-state lifetime $(1.3 \mathrm{~ms})$.

In Fig. 2(a), the absorption line shape does not change with pumping duration, and the fraction of pumped ions is small. The peak absorption coefficient measured at $0 \mathrm{GHz}$ and normalized by its value without OP, i.e., at thermal equilibrium, is therefore equivalent to $k_{4 g}$.

\section{Optical coherence measurement}

For those measurements, a second AOM was added to enhance gating and avoid optical pumping during photon echo sequences. The beam was focused by a $100-\mathrm{mm}$ focal length lens with a power of $7 \mathrm{~mW}$. The photon echo was measured using a standard Hahn echo sequence $(\pi / 2-\tau-\pi-\tau-$ echo) with durations of 1 and $2 \mu \mathrm{s}$ for the $\pi / 2$ and $\pi$ pulses. Because of the laser jitter, the echo amplitude significantly fluctuated for $\tau>300 \mu \mathrm{s}$. To overcome this issue, for a given delay $\tau, 50$ successive echo sequences were recorded, and only the strongest echo was kept. We checked that the echo sequences themselves did not cause optical pumping. Echo pulse power was also varied to look for instantaneous spectral diffusion, which was not observed.

[1] M. Atatüre, D. Englund, N. Vamivakas, S.-Y. Lee, and J. Wrachtrup, Material Platforms for Spin-Based Photonic Quantum Technologies, Nat. Rev. Mater. 3, 38 (2018).

[2] F. Bussières, C. Clausen, A. Tiranov, B. Korzh, V. B. Verma, S. W. Nam, F. Marsili, A. Ferrier, P. Goldner, H. Herrmann, C. Silberhorn, W. Sohler, M. Afzelius, and N. Gisin, Quantum Teleportation from a Telecom-Wavelength Photon to a Solid-State Quantum Memory, Nat. Photonics 8, 775 (2014).

[3] B. Hensen, H. Bernien, A. E. Dréau, A. Reiserer, N. Kalb, M. S. Blok, J. Ruitenberg, R. F. L. Vermeulen, R. N. Schouten, C. Abellán, W. Amaya, V. Pruneri, M. W. Mitchell, M. Markham, D. J. Twitchen, D. Elkouss, S. Wehner, T. H. Taminiau, and R. Hanson, Loophole-Free Bell Inequality Violation Using Electron Spins Separated by 1.3 Kilometres, Nature (London) 526, 682 (2015).

[4] H. J. Kimble, The Quantum Internet, Nature (London) 453, 1023 (2008). 
[5] J. M. Boss, K. S. Cujia, J. Zopes, and C. L. Degen, Quantum Sensing with Arbitrary Frequency Resolution, Science 356, 837 (2017).

[6] M. Zhong, M. P. Hedges, R. L. Ahlefeldt, J. G. Bartholomew, S. E. Beavan, S. M. Wittig, J. J. Longdell, and M. J. Sellars, Optically Addressable Nuclear Spins in a Solid with a SixHour Coherence Time, Nature (London) 517, 177 (2015).

[7] D. Serrano, J. Karlsson, A. Fossati, A. Ferrier, and P. Goldner, All-Optical Control of Long-Lived Nuclear Spins in Rare-Earth Doped Nanoparticles, Nat. Commun. 9, 2127 (2018).

[8] T. Böttger, C. W. Thiel, Y. Sun, and R. L. Cone, Optical Decoherence and Spectral Diffusion at $1.5 \mu \mathrm{m}$ in $\mathrm{Er}^{3+}: \mathrm{Y}_{2} \mathrm{SiO}_{5}$ versus Magnetic Field, Temperature, and $\mathrm{Er}^{3+}$ Concentration, Phys. Rev. B 73, 075101 (2006).

[9] H. S. Knowles, D. M. Kara, and M. Atatüre, Observing Bulk Diamond Spin Coherence in High-Purity Nanodiamonds, Nat. Mater. 13, 21 (2014).

[10] G. Balasubramanian, P. Neumann, D. Twitchen, M. Markham, R. Kolesov, N. Mizuochi, J. Isoya, J. Achard, J. Beck, J. Tissler, V. Jacques, P. R. Hemmer, F. Jelezko, and J. Wrachtrup, Ultralong Spin Coherence Time in Isotopically Engineered Diamond, Nat. Mater. 8, 383 (2009).

[11] A. M. Tyryshkin, S. Tojo, J. J. L. Morton, H. Riemann, N. V. Abrosimov, P. Becker, H.-J. Pohl, T. Schenkel, M. L. W. Thewalt, K. M. Itoh, and S. A. Lyon, Electron Spin Coherence Exceeding Seconds in High-Purity Silicon, Nat. Mater. 11, 143 (2012).

[12] T. Böttger, C. W. Thiel, R. L. Cone, and Y. Sun, Effects of Magnetic Field Orientation on Optical Decoherence in $\mathrm{Er}^{3+}: \mathrm{Y}_{2} \mathrm{SiO}_{5}$, Phys. Rev. B 79, 115104 (2009).

[13] M. Rancic, M. P. Hedges, R. L. Ahlefeldt, and M. J. Sellars, Coherence Time of over a Second in a Telecom-Compatible Quantum Memory Storage Material, Nat. Phys. 14, 50 (2018).

[14] N. Kukharchyk, D. Sholokhov, O. Morozov, S. L. Korableva, A. A. Kalachev, and P. A. Bushev, Optical Coherence of ${ }^{166} \mathrm{Er}:{ }^{7} \mathrm{LiYF}_{4}$ Crystal below $1 \mathrm{~K}$, New J. Phys. 20, 023044 (2018).

[15] T. Chakraborty, J. Zhang, and D. Suter, Polarizing the Electronic and Nuclear Spin of the NV-Center in Diamond in Arbitrary Magnetic Fields: Analysis of the Optical Pumping Process, New J. Phys. 19, 073030 (2017).

[16] E. Fraval, M. J. Sellars, and J. J. Longdell, Method of Extending Hyperfine Coherence Times in $\mathrm{Pr}^{3+}: \mathrm{Y}_{2} \mathrm{SiO}_{5}$, Phys. Rev. Lett. 92, 077601 (2004).

[17] G. Wolfowicz, A. M. Tyryshkin, R. E. George, H. Riemann, N. V. Abrosimov, P. Becker, H.-J. Pohl, M. L. W. Thewalt, S. A. Lyon, and J. J. L. Morton, Atomic Clock Transitions in Silicon-Based Spin Qubits, Nat. Nanotechnol. 8, 561 (2013).

[18] A. Ortu, A. Tiranov, S. Welinski, F. Fröwis, N. Gisin, A. Ferrier, P. Goldner, and M. Afzelius, Simultaneous Coherence Enhancement of Optical and Microwave Transitions in Solid-State Electronic Spins, Nat. Mater. 17, 671 (2018).

[19] L. Viola and S. Lloyd, Dynamical Suppression of Decoherence in Two-State Quantum Systems, Phys. Rev. A 58, 2733 (1998).
[20] M. Lovrić, D. Suter, A. Ferrier, and P. Goldner, Faithful Solid State Optical Memory with Dynamically Decoupled Spin Wave Storage, Phys. Rev. Lett. 111, 020503 (2013).

[21] P. Goldner, A. Ferrier, and O. Guillot-Noël, Rare EarthDoped Crystals for Quantum Information Processing, in Handbook on the Physics and Chemistry of Rare Earths, edited by J.-C. G. Bünzli and V. K. Pecharsky (Elsevier, Amsterdam, 2015), pp. 1-78.

[22] E. Saglamyurek, N. Sinclair, J. Jin, J. A. Slater, D. Oblak, F. Bussières, M. George, R. Ricken, W. Sohler, and W. Tittel, Broadband Waveguide Quantum Memory for Entangled Photons, Nature (London) 469, 512 (2011).

[23] Z.-Q. Zhou, W.-B. Lin, M. Yang, C.-F. Li, and G.-C. Guo, Realization of Reliable Solid-State Quantum Memory for Photonic Polarization Qubit, Phys. Rev. Lett. 108, 190505 (2012).

[24] T. Zhong, J. M. Kindem, J. G. Bartholomew, J. Rochman, I. Craiciu, E. Miyazono, M. Bettinelli, E. Cavalli, V. Verma, S. W. Nam, F. Marsili, M. D. Shaw, A. D. Beyer, and A. Faraon, Nanophotonic Rare-Earth Quantum Memory with Optically Controlled Retrieval, Science 357, 1392 (2017).

[25] C. Laplane, P. Jobez, J. Etesse, N. Gisin, and M. Afzelius, Multimode and Long-Lived Quantum Correlations between Photons and Spins in a Crystal, Phys. Rev. Lett. 118, 210501 (2017).

[26] A. Walther, L. Rippe, Y. Yan, J. Karlsson, D. Serrano, A. N. Nilsson, S. Bengtsson, and S. Kröll, High-Fidelity Readout Scheme for Rare-Earth Solid-State Quantum Computing, Phys. Rev. A 92, 022319 (2015).

[27] A. M. Dibos, M. Raha, C. M. Phenicie, and J. D. Thompson, Atomic Source of Single Photons in the Telecom Band, Phys. Rev. Lett. 120, 243601 (2018).

[28] T. Zhong, J. M. Kindem, J. G. Bartholomew, J. Rochman, I. Craiciu, V. Verma, S. W. Nam, F. Marsili, M. D. Shaw, A. D. Beyer, and A. Faraon, Optically Addressing Single Rare-Earth Ions in a Nanophotonic Cavity, Phys. Rev. Lett. 121, 183603 (2018).

[29] L. A. Williamson, Y.-H. Chen, and J. J. Longdell, MagnetoOptic Modulator with Unit Quantum Efficiency, Phys. Rev. Lett. 113, 203601 (2014).

[30] S. Welinski, P. J. T. Woodburn, N. Lauk, R. L. Cone, C. Simon, P. Goldner, and C. W. Thiel, Electron Spin Coherence in Optically Excited States of Rare-Earth Ions for Microwave to Optical Quantum Transducers, Phys. Rev. Lett. 122, 247401 (2019).

[31] S. Welinski, A. Ferrier, M. Afzelius, and P. Goldner, HighResolution Optical Spectroscopy and Magnetic Properties of $\mathrm{Yb}^{3+}$ in $\mathrm{Y}_{2} \mathrm{SiO}_{5}$, Phys. Rev. B 94, 155116 (2016).

[32] A. Tiranov, A. Ortu, S. Welinski, A. Ferrier, P. Goldner, N. Gisin, and M. Afzelius, Spectroscopic Study of Hyperfine Properties in ${ }^{171} \mathrm{Yb}^{3+}: \mathrm{Y}_{2} \mathrm{SiO}_{5}$, Phys. Rev. B 98, 195110 (2018).

[33] H.-J. Lim, S. Welinski, A. Ferrier, P. Goldner, and J. J. L. Morton, Coherent Spin Dynamics of Ytterbium Ions in Yttrium Orthosilicate, Phys. Rev. B 97, 064409 (2018).

[34] J. M. Kindem, J. G. Bartholomew, P. J. T. Woodburn, T. Zhong, I. Craiciu, R. L. Cone, C. W. Thiel, and A. Faraon, Characterization of ${ }^{171} \mathrm{Yb}^{3+}: \mathrm{YVO}_{4}$ for Photonic Quantum Technologies, Phys. Rev. B 98, 024404 (2018). 
[35] P. E. Jessop and A. Szabo, Optical Hole-Burning and Ground State Energy Transfer in Ruby, in Laser Spectroscopy V (Springer, Berlin, Heidelberg, 1981), pp. 408-411.

[36] R. L. Ahlefeldt, M. R. Hush, and M. J. Sellars, Ultranarrow Optical Inhomogeneous Linewidth in a Stoichiometric Rare-Earth Crystal, Phys. Rev. Lett. 117, 250504 (2016).

[37] M. J. Sellars, P. T. H. Fisk, and N. B. Manson, Long-Time Spectral Diffusion in $\mathrm{LaF}_{3}: \operatorname{Pr}^{3+}$, J. Lumin. 58, 188 (1994).

[38] See Supplemental Material at [http://link.aps.org/ supplemental/10.1103/PhysRevX.10.031060] for further details about ground-state population measurement, DEOP modeling, spin coherence time measurements, polarization into a single hyperfine level, DEOP in other materials, and DEOP for quantum memories and optical to microwave transducers.

[39] R. M. Macfarlane and R. M. Shelby, Coherent Transient and Hole Burning Spectroscopy of Rare Earth Ions in Solids, in Spectroscopy of Solids Containing Rare Earth Ions, edited by A. A. Kaplyanskii and R. M. Macfarlane (North-Holland, Amsterdam, 1987), pp. 51-184.

[40] M. Businger, A. Tiranov, K. T. Kaczmarek, S. Welinski, Z. Zhang, A. Ferrier, P. Goldner, and M. Afzelius, Optical Spin-Wave Storage in a Solid-State Hybridized ElectronNuclear Spin Ensemble, Phys. Rev. Lett. 124, 053606 (2020).

[41] A. M. Stoneham, Shapes of Inhomogeneously Broadened Resonance Lines in Solids, Rev. Mod. Phys. 41, 82 (1969).

[42] S. Probst, H. Rotzinger, A. V. Ustinov, and P. A. Bushev, Microwave Multimode Memory with an Erbium Spin Ensemble, Phys. Rev. B 92, 014421 (2015).

[43] E. Z. Cruzeiro, A. Tiranov, I. Usmani, C. Laplane, J. Lavoie, A. Ferrier, P. Goldner, N. Gisin, and M. Afzelius, Spectral Hole Lifetimes and Spin Population Relaxation Dynamics in Neodymium-Doped Ytrium Orthosilicate, Phys. Rev. B 95, 205119 (2017).

[44] R. W. Equall, Y. Sun, R. L. Cone, and R. M. Macfarlane, Ultraslow Optical Dephasing in $\mathrm{Eu}^{3+}: \mathrm{Y}_{2} \mathrm{SiO}_{5}$, Phys. Rev. Lett. 72, 2179 (1994).

[45] Y. C. Sun, Rare Earth Materials in Optical Storage and Data Processing Applications, in Spectroscopic Properties of Rare Earths in Optical Materials, edited by G. Liu and B. Jacquier (Springer, Berlin, 2005), pp. 379-429.

[46] J. J. Longdell, A. Alexander, and M. J. Sellars, Characterization of the Hyperfine Interaction in Europium-Doped Yttrium Orthosilicate and Europium Chloride Hexahydrate, Phys. Rev. B 74, 195101 (2006).

[47] B. Casabone, J. Benedikter, T. Hümmer, F. Oehl, K. de Oliveira Lima, T. W. Hänsch, A. Ferrier, P. Goldner, H. de Riedmatten, and D. Hunger, Cavity-Enhanced Spectroscopy of a Few-Ion Ensemble in $\mathrm{Eu}^{3+}: \mathrm{Y}_{2} \mathrm{O}_{3}$, New J. Phys. 20, 095006 (2018).

[48] R. T. Harley, M. J. Henderson, and R. M. Macfarlane, Persistent Spectral Hole Burning of Colour Centres in Diamond, J. Phys. C 17, L233 (2000).

[49] M. W. Doherty, N. B. Manson, P. Delaney, F. Jelezko, J. Wrachtrup, and L. C. L. Hollenberg, The Nitrogen-Vacancy Colour Centre in Diamond, Phys. Rep. 528, 1 (2013).
[50] T. Böttger, Y. Sun, C. W. Thiel, and R. L. Cone, Spectroscopy and Dynamics of $\mathrm{Er}^{3+}: \mathrm{Y}_{2} \mathrm{SiO}_{5}$ at $1.5 \mu \mathrm{m}$, Phys. Rev. B 74, 075107 (2006).

[51] M. Afzelius, M. U. Staudt, H. de Riedmatten, N. Gisin, O. Guillot-Noël, P. Goldner, R. Marino, P. Porcher, E. Cavalli, and M. Bettinelli, Efficient Optical Pumping of Zeeman Spin Levels in $\mathrm{Nd}^{3+}: \mathrm{YVO}_{4}$, J. Lumin. 130, 1566 (2010).

[52] E. Z. Cruzeiro, A. Tiranov, J. Lavoie, A. Ferrier, P. Goldner, N. Gisin, and M. Afzelius, Efficient Optical Pumping Using Hyperfine Levels in ${ }^{145} \mathrm{Nd}^{3+}: \mathrm{Y}_{2} \mathrm{SiO}_{5}$ and Its Application to Optical Storage, New J. Phys. 20, 053013 (2018).

[53] X. Fernandez-Gonzalvo, S. P. Horvath, Y.-H. Chen, and J. J. Longdell, Cavity-Enhanced Raman Heterodyne Spectroscopy in $\mathrm{Er}^{3+}: \mathrm{Y}_{2} \mathrm{SiO}_{5}$ for Microwave to Optical Signal Conversion, Phys. Rev. A 100, 033807 (2019).

[54] M. Raha, S. Chen, C. M. Phenicie, S. Ourari, A. M. Dibos, and J.D. Thompson, Optical Quantum Nondemolition Measurement of a Single Rare Earth Ion Qubit, Nat. Commun. 11, 1605 (2020).

[55] J. V. Rakonjac, Y.-H. Chen, S. P. Horvath, and J. J. Longdell, Long Spin Coherence Times in the Ground State and in an Optically Excited State of ${ }^{167} \mathrm{Er}^{3+}: \mathrm{Y}_{2} \mathrm{SiO}_{5}$ at Zero Magnetic Field, Phys. Rev. B 101, 184430 (2020).

[56] D. D. Awschalom, R. Hanson, J. Wrachtrup, and B. B. Zhou, Quantum Technologies with Optically Interfaced Solid-State Spins, Nat. Photonics 12, 516 (2018).

[57] C. Bradac, W. Gao, J. Forneris, M. E. Trusheim, and I. Aharonovich, Quantum Nanophotonics with Group IV Defects in Diamond, Nat. Commun. 10, 5625 (2019).

[58] K. J. Morse, R. J. S. Abraham, A. DeAbreu, C. Bowness, T. S. Richards, H. Riemann, N. V. Abrosimov, P. Becker, H.-J. Pohl, M. L. W. Thewalt, and S. Simmons, A Photonic Platform for Donor Spin Qubits in Silicon, Sci. Adv. 3, e1700930 (2017).

[59] F. Bussières, N. Sangouard, M. Afzelius, H. de Riedmatten, C. Simon, and W. Tittel, Prospective Applications of Optical Quantum Memories, J. Mod. Opt. 60, 1519 (2013).

[60] V. Damon, M. Bonarota, A. Louchet-Chauvet, T. Chanelière, and J.-L. Le Gouët, Revival of Silenced Echo and Quantum Memory for Light, New J. Phys. 13, 093031 (2011).

[61] A. Arcangeli, A. Ferrier, and P. Goldner, Stark Echo Modulation for Quantum Memories, Phys. Rev. A 93, 062303 (2016).

[62] S. E. Beavan, M. P. Hedges, and M. J. Sellars, Demonstration of Photon-Echo Rephasing of Spontaneous Emission, Phys. Rev. Lett. 109, 093603 (2012).

[63] H. de Riedmatten, M. Afzelius, M. U. Staudt, C. Simon, and N. Gisin, A Solid-State Light-Matter Interface at the SinglePhoton Level, Nature (London) 456, 773 (2008).

[64] K. Debnath, A. H. Kiilerich, A. Benseny, and K. Mølmer, Coherent Spectral Hole Burning and Qubit Isolation by Stimulated Raman Adiabatic Passage, Phys. Rev. A 100, 023813 (2019).

[65] C. O'Brien, N. Lauk, S. Blum, G. Morigi, and M. Fleischhauer, Interfacing Superconducting Qubits and Telecom Photons via a Rare-Earth-Doped Crystal, Phys. Rev. Lett. 113, 063603 (2014). 